BI-TP 99/32

September 1999

\title{
Further Evidence for an unstable H-Dibaryon?
}

\author{
I. Wetzorke with F. Karsch, E. Laermann * \\ Fakultät für Physik, Universität Bielefeld, D-33615 Bielefeld, Germany
}

\begin{abstract}
We present preliminary results for the mass of the 6q flavor-singlet state $\left(J^{P}=0^{+}, S=-2\right)$ called H-dibaryon, calculated in quenched QCD on $16^{3} \times 30$ and $24^{3} \times 30$ lattices with improved gauge and fermion actions (Symanzik improvement, Clover action). For both lattice sizes we applied the fuzzing technique to enhance the overlap with the ground state. We observe a $\mathrm{H}$-mass above the $\Lambda \Lambda$-threshold for strong decay. The difference in mass, $m_{H}-2 m_{\Lambda}$, increases with increasing lattice size.
\end{abstract}

\section{INTRODUCTION}

In 1977 a bound six-quark state (uuddss), the $\mathrm{H}$-dibaryon, was predicted in a bag-model calculation by Jaffe [1]. This state is the lowest $\mathrm{SU}(3)$ flavor singlet state with spin zero, strangeness -2 and $J^{P}=0^{+}$. These dibaryons may play an important role as Bose condensate in nuclear matter prior to the quark-hadron phase transition of QCD at high density.

In the last twenty years many attempts to verify the existence and stability of this particle were undertaken by means of various methods. Perturbative calculations included spin-dependent $\mathrm{q}-\mathrm{q}$ interactions arising from one gluon exchange (OGE) $[1,2]$, instanton induced interactions (III) $[3,4]$ and Goldstone boson exchange (GBE) [5]. The attractive $\mathrm{q}-\mathrm{q}$ interaction is controlled via the potential $V_{s} \sim\left(\lambda_{1}^{a} \lambda_{2}^{a}\right)\left(s_{1} s_{2}\right)$, where $\lambda_{i}^{a}$ denote $\mathrm{SU}(3)$ color (OGE) or flavor (III/GBE) generators. The resulting $\mathrm{H}$-masses scattered in a range of a few hundred $\mathrm{MeV}$ around the $2231 \mathrm{MeV}$ $\Lambda \Lambda$-threshold for strong decay.

The lattice attempts to calculate $m_{H}$ a decade ago $[6,7]$ gave contradicting results, but a new calculation [8] showed that the q-q attraction decreases with increasing volume. Hence it has been suggested that the H-dibaryon is unbound (approx. $110 \mathrm{MeV}$ above the threshold) in the infinite volume extrapolation.

\footnotetext{
*The work has been supported by the TMR network ERBFMRX-CT-970122 and the DFG under grant Ka 1198/4-1.
}

\section{DETAILS OF THE SIMULATION}

Our spectrum calculation was performed in quenched QCD with Wilson fermions on lattices of the size $16^{3} \times 30$ and $24^{3} \times 30$. We generated 57 independent gauge field configurations for the smaller lattice and 15 configurations so far for the larger lattice, separated by 100 sweeps of 4 overrelaxation and one heatbath step each. For the gauge sector we applied the tree-level improved $(1,2)$ Symanzik action at a gauge coupling $\beta=4.1$, which corresponds to a lattice spacing $a=0.177(8)$ fm defined by the string tension [9]. A tree-level improved clover term was used in the fermionic part of the action.

The correlation functions were calculated via the relation $C(t)=\left\langle\mathcal{O}(0) \mathcal{O}^{\dagger}(t)\right\rangle$ with appropriate operators for the lambda and the H-dibaryon [10] (color indices: roman letters, spinor indices: greek letters):

$$
\begin{gathered}
\mathcal{O}_{\Lambda}(x)=\epsilon_{a b c}\left(C \gamma_{5}\right)_{\beta \gamma}\left[u_{\alpha}^{a}(x) d_{\beta}^{b}(x) s_{\gamma}^{c}(x)\right. \\
\left.+d_{\alpha}^{a}(x) s_{\beta}^{b}(x) u_{\gamma}^{c}(x)-2 s_{\alpha}^{a}(x) u_{\beta}^{b}(x) d_{\gamma}^{c}(x)\right] \\
\mathcal{O}_{H}(x)=3(u d s u d s)-3(u s s u d d)-3(d s s d u u) \\
(\text { abcdef })=\epsilon_{a b c} \epsilon_{d e f}\left(C \gamma_{5}\right)_{\alpha \beta}\left(C \gamma_{5}\right)_{\gamma \delta}\left(C \gamma_{5}\right)_{\epsilon \phi} \\
\quad * \mathrm{a}_{\alpha}^{a}(x) \mathbf{b}_{\beta}^{b}(x) \mathbf{c}_{\epsilon}^{c}(x) \mathrm{d}_{\gamma}^{d}(x) \mathrm{e}_{\delta}^{e}(x) f_{\phi}^{f}(x)
\end{gathered}
$$

We summed over spatial coordinates to project onto zero momentum and over all spinor and color indices to get diagonal correlators.

Quark propagators were calculated at five different values of the bare quark mass, which have been estimated to be in the range $50-250 \mathrm{MeV}$. 


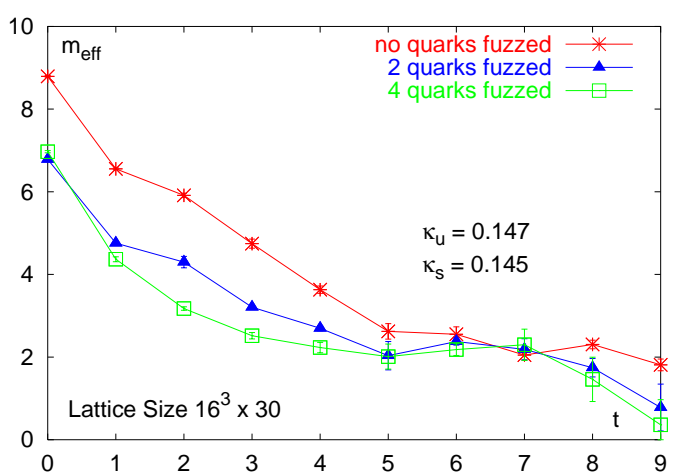

Figure 1. Influence of fuzzing on the dibaryon correlation function through different number of fuzzed quark propagators

The mass is controlled through the hopping parameter $\kappa_{u}$ for the degenerated $\mathrm{u}$ - and d-quark mass and $\kappa_{s}$ for the s-quark mass. The physical $\kappa_{u}$ used in the extrapolation was set by the ratio of nucleon mass and string tension. The value for $\kappa_{s}$ was determined by the mass ratio of lambda and nucleon.

In addition to the H-dibaryon and lambda we calculated the correlation functions for the strange mesons $K, K^{*}$ and the $\Sigma$ baryon. The obtained particle masses lie all in a $10 \%$ range around the experimental values, which is a common result in calculations using the quenched approximation.

\section{FUZZING}

Extended operators and smeared gauge fields provide a better overlap with the ground state [11]. For the quark fields we use:

$$
\begin{array}{r}
\Psi^{\prime}(x, R)=\sum_{\mu \in V_{z}}\left(U^{\dagger}(x-\hat{\mu}) \ldots U^{\dagger}(x-R \hat{\mu}) \Psi(x-R \hat{\mu})\right. \\
+U(x) \ldots U(x+(R-1) \hat{\mu}) \Psi(x+R \hat{\mu}))
\end{array}
$$

Smeared gauge fields improve the simulation of the gluon cloud around the quarks:

$U^{\prime}(x, \mu)=\mathcal{P}_{S U(3)}\left[c U(x, \mu)+\sum_{ \pm \nu \neq \mu<4} U_{\text {staples }(\nu)}\right]$

The application of fuzzing for two of the six quarks inside the dibaryon flattens the curvature of the effective mass (Fig. 1). The largest plateau

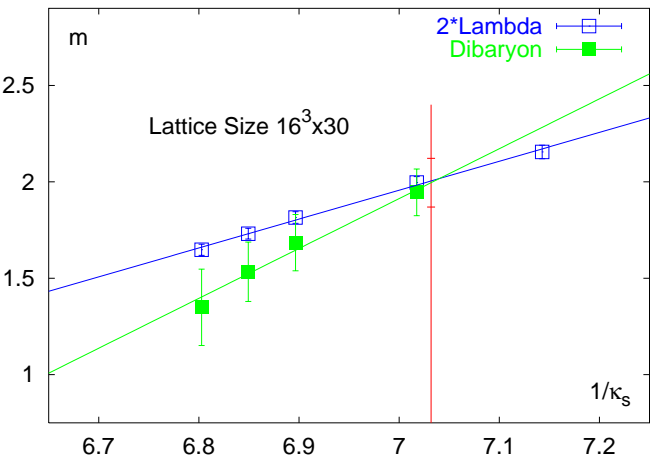

Figure 2. Lambda and H-masses extrapolated to the physical $\kappa_{u}=0.149$ (line indicates physical $\kappa_{s}$ )

in the region with small errors is obtained with fuzzed quarks for the four lighter u- and d-quarks. Therefore we used this variant to calculate our correlation functions.

\section{RESULTS}

\subsection{Comparison of Dibaryon and Lambda Masses}

The particle masses were extracted from the long range behavior of the correlation functions. We leave out successively data points in the exponential fits at small t to yield stable results. Contributions of excited states were substantially reduced by the fuzzing technique. Additionally, fits with two exponentials enlarge the plateau even for the first time slices.

The results on the smaller lattice show a H-mass in the same range as twice the $\Lambda$-mass (Fig. 2). We obtained $m_{H}=2221(141) \mathrm{MeV}$, which is just below the $\Lambda \Lambda$-threshold for strong decay. For the difference in mass we obtain $m_{H}-2 m_{\Lambda}=$ $-10 \pm 141 \mathrm{MeV}$. It is therefore impossible to decide, whether the dibaryon is unbound or slightly bound. Moreover, we cannot rule out considerable finite size effects on the small $(2.8 \mathrm{fm})^{3}$ lattice.

The mass splitting on the larger lattice $(4.2 \mathrm{fm})^{3}$ seems to be more pronounced (Fig. 3). The Hmass is larger than two $\Lambda$ 's for the parameter combinations calculated so far. Although our 


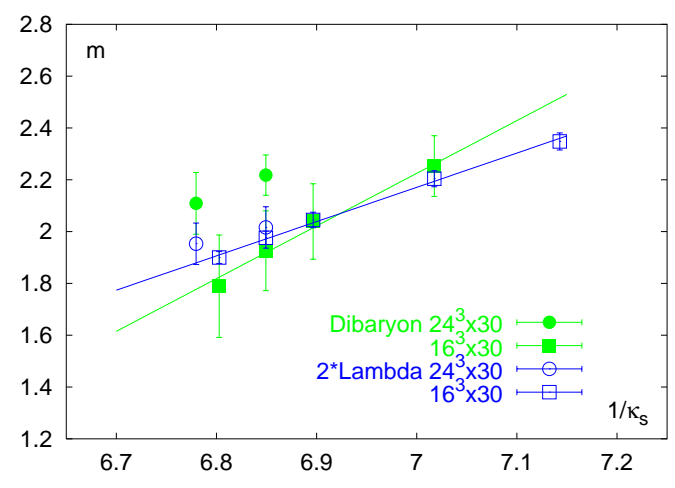

Figure 3. Lambda and dibaryon masses on both lattices for $\kappa_{u}=0.1475$

statistics on the large lattice is not yet sufficient to draw a definite conclusion, the preliminary results shown in Fig. 3 suggest that the H-dibaryon is unbound in the infinite volume limit.

\subsection{Ratio of the Correlation Functions}

The errors in the correlation functions for the dibaryon and the lambda are strongly correlated. Some of the statistical uncertainties can thus be eliminated by analysing directly the ratio of correlation functions. The ratio of the correlation functions of dibaryon and lambda should rise, when $m_{H}<2 m_{\Lambda}$ :

$C(H) \sim e^{-m_{H} t} \Longrightarrow \frac{C(H)}{C^{2}(\Lambda)} \sim e^{\left(2 m_{\Lambda}-m_{H}\right) t}$,

or should be constant, when $m_{H}>2 m_{\Lambda}$ :

$C(H) \sim e^{-2 m_{\Lambda} t} \Longrightarrow \frac{C(H)}{C^{2}(\Lambda)} \sim$ const.

On the smaller lattice this ratio is rising at $t \geq 8$ with and without fuzzing (Fig. 4), which provides evidence of a slightly bound H-dibaryon. On the larger lattice the ratio remains constant until $t=8$. With our present statistics the signal gets lost for larger values of $t$. Nonetheless, the large plateau of constant values again suggests that we see an unbound H-dibaryon on this larger lattice.

\section{CONCLUSIONS \& OUTLOOK}

With our present, still preliminary, analysis of the H-dibaryon and $\Lambda$ masses we confirm the find-

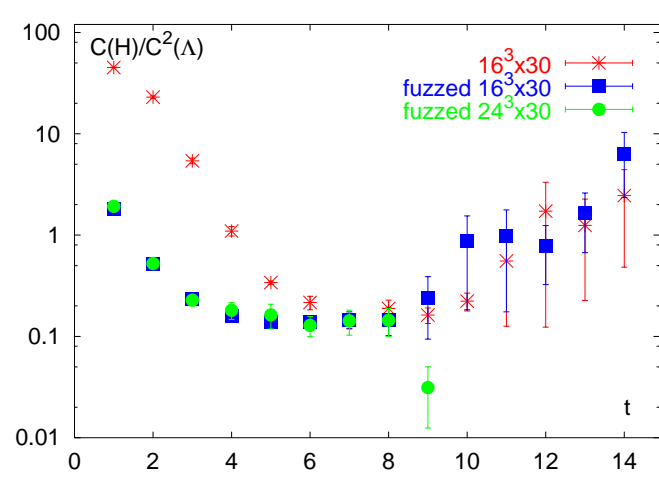

Figure 4. Ratio of the correlation functions for $\kappa_{u}=0.147\left(0.1475\right.$ for $\left.24^{3} \times 30\right)$ and $\kappa_{s}=0.146$

ings of Negele et al. [8] presented at last year's lattice conference. We observe that the difference in mass, $m_{H}-2 m_{\Lambda}$, increases with increasing lattice size and therefore will lead to an unbound $\mathrm{H}$-dibaryon in the infinite volume limit.

We will continue our simulations on the larger lattice with more configurations and additional combinations of $\kappa_{u}$ and $\kappa_{s}$ to extrapolate to the physical values of the masses. Simulations on a smaller lattice would be advantageous to confirm the connection between finite lattice size and the size of the mass splitting $m_{H}-2 m_{\Lambda}$.

\section{REFERENCES}

1. R. L. Jaffe, Phys. Rev. Lett. 38 (1977) 195

2. K. Maltman et al., Phys. Lett. B393 (1997) 274 and Mod. Phys. Lett. A13 (1998) 59

3. S. Takeuchi and M. Oka, Phys. Rev. Lett. 66 (1991) 1271

4. N. I. Kochelev, hep-ph/9905333

5. Fl. Stancu et al., Phys. Rev. D57 (1998) 4393

6. P. B. Mackenzie, H. B. Thacker, Phys. Rev. Lett. 55 (1985) 2539

7. Y. Iwasaki et al., Phys. Rev. Lett. 60 (1988) 1371

8. J. W. Negele et al., Nucl. Phys. Proc. Suppl. 73 (1999) 255

9. B. Beinlich et al., Eur. Phys. J. C6 (1999) 133

10. E. Golowich and T. Sotirelis, Phys. Rev. D46 (1992) 354

11. UKQCD: P. Lacock et al., Phys. Rev. D51 (1995) 6403 